- Co-ordinating the WP-rota takes commitment and personal time for the trainee lead - we suggest allocating 4 hours' admin time each fortnight for this.

- Taking on this role improves a trainee's leadership and management experience.

\section{G394(P) CAMPHOR INGESTION - A RARE CAUSE OF ACUTE SEIZURE ATTACK}

P Kadambi, MA Ramprakash, DLK Anusha, G Sowjanya. Dept. of Paediatrics, Meenakshi Medical College Hospital and Research Centre, Kanchipuram, India

\subsection{6/archdischild-2020-rcpch.338}

Introduction Camphor is a toxic compound which is available freely over-the-counter (OTC) with potential to cause fatal seizures in paediatric age group when ingested. The lethal dose of camphor is $30 \mathrm{mg} / \mathrm{kg}$. It is available in many Indian households as it is used for day to day religious rituals. In Indian context, there is limited data with respect to incidence, prevalence of camphor ingestion related seizures and its associated complications.

Case Report A 2 year old male child, second born to third degree consanguineously married couple completely immunized with known speech delay was brought to the emergency department with history of jerky movements of Right Upper Limb and 2 episodes of projectile vomiting four hours preceding arrival. On arrival, his airway was patent and vital signs were stable. His father gave a history of jerky movements of right upper limb while playing after which the child fell backwards followed by a 5 minute loss of consciousness. This was followed by 2 episodes of projectile vomiting non-blood tinged, non-bile stained. Neurological examination was unremarkable. Child was admitted in PICU and started on loading dose phenytoin $(20 \mathrm{mg} / \mathrm{kg} / \mathrm{day})$ followed by maintenance ( $5 \mathrm{mg} / \mathrm{kg} /$ day $) .24$ hours post-admission, first fever was recorded and second episode of seizure recurred, noted to be of Generalised tonic clonic type. Tab. Clobazam $5 \mathrm{mg}$ was added prophylactically. Lumbar puncture was done and reports within normal limits. Electroencephalogram showed generalized brain dysfunction with epileptic potentials. MRI Brain was normal. Upon enquiring the history again with the parents, they revealed a history of camphor ingestion (2 tablets -approximately 10 grams) on the day of seizures which they intentionally concealed initially considering it to be irrelevant. The child was discharged on oral maintenance phenytoin and came back for follow-up after a week at which all medications were stopped. At 3rd week follow up, the child is doing well with no seizure activities.

Conclusion Camphor ingestion is a rare, preventable cause of seizures. This case also re-emphasizes upon the importance of clinical history. There is also a need for centralized toxicology database for reporting such cases for benefit to the clinical community.

\section{G395(P) MANAGEMENT OF A CASE OF SEVERE NEONATAL PNEUMONIA BY INDIGENOUS BUBBLE CPAP}

P Kadambi, MA Ramprakash. Dept of Paediatrics, Meenakshi Medical College Hospital and Research Centre, Kanchipuram, India

10.1136/archdischild-2020-rcpch.339
Introduction Continuous Positive Airway Pressure (CPAP) is a commonly employed technique in critical care setups. Indigenously developed low cost CPAP devices are helpful in reducing the need of mechanical ventilation and reduction of mortality while maintaining the standards of care.

Case Report A 26 day male neonate was brought to the outpatient department with complaints of cough and cold since 4 days, history of breathlessness and history of refusal of feeds from 1 day. On examination, respiratory rate was 70/min, oxygen saturation $92 \%$ at room air, work of breathing increased with distress and retractions. Baby was admitted under NICU and sepsis investigations done. Baby was started on IV fluids, Inj. Amoxyclav (50 mg/kg/day), Inj Amikacin $\left(150 \mathrm{mg} / \mathrm{kg} /\right.$ day) and on $4 \mathrm{~L} \mathrm{O}_{2}$ thru nasal prongs. However, oxygen saturation continued to drop to $88 \%$. Baby was started on indigenous bubble Continuous Positive Airway Pressure (CPAP) and feeds given as Expressed Breastmilk thru orogastric tube $(10 \mathrm{ml} / 2 \mathrm{hrs})$. On Day 3, orogastric tube was removed and supervised EBM paladey feeds given. On Day 4, IV Fluids tapered and stopped while direct breastfeeds initiated under supervision. On Day 5, CPAP removed and kept on $\mathrm{O}_{2}$ thru nasal prongs at $1 / 2 \mathrm{~L} \mathrm{O}_{2}$. Trial to wean off $\mathrm{O}_{2}$ attempted on Day 6 but not successful. Repeat X-Ray done and $\mathrm{O}_{2}$ weaned on Day 7. Baby shifted to ward and kept under observation. Baby was discharged on Day 8 and came for follow up and is doing well.

Conclusion Our case highlights the efficacy of Indigenous bubble CPAP in management of respiratory distress preventing need for mechanical ventilation and the potential for use of this technique on large scale in low-resource setups. However, this can be done after validation by studies on large scale.

\section{G396(P) A QUANTITATIVE AND QUALITATIVE ASSESSMENT OF SLEEP IN CHILDREN AGED 3-15 YEARS: A PILOT STUDY}

P Kadambi, SC Nivedhidha. Dept of Paediatrics, Meenakshi Medical College Hospital and Research Centre, Kanchipuram, India

\subsection{6/archdischild-2020-rcpch.340}

Introduction Sleep plays a vital role in good health, growth and well-being. Sleep disorders manifest with disturbance in quantity and quality. Previous studies have shown that sleep disorders are increasing in pediatric age group, which is attributed to increased usage of smartphones and also associated with lifestyle changes.

Aim To assess quality and quantity of sleep in children aged 3-15 years.

Methodology Institutional Ethics Committee approval was obtained. 52 children were recruited for the study from Meenakshi Medical College Hospital, Kanchipuram, India. After obtaining consent, Pediatric Sleep Questionnaire (PSQ) was administered after collecting clinical history. History of duration of smartphone usage, TV Screen time, history of hospitalizations, time spent outdoors was also collected. Anthropometry and clinical examination was done. Data was tabulated and analysed using SPSS V17.0.

Results 4 out of 52 children (7.69\%) in the study had poor quality sleep (PSQ Score $\geq 5$ ). 24 out of 52 children $(46.1 \%$ ) had deficient quantity of sleep as per WHO recommendations. Children born with low birth weight had comparatively poorer quality of sleep $(\mathrm{r}=0.331 \mathrm{p}=0.015)$. 